\title{
Broadband excitation spectrum of bulk crystals and thin layers of $\mathrm{PtTe}_{2}$
}

\author{
Barun Ghosh, ${ }^{1}$ Francesca Alessandro, ${ }^{2,3}$ Marilena Zappia, ${ }^{2}$ Rosaria Brescia,${ }^{4}$ Chia-Nung Kuo, ${ }^{5}$ Chin Shan \\ Lue ${ }^{5}$ Gennaro Chiarello, ${ }^{2}$ Antonio Politano, ${ }^{6,7}$ Lorenzo S. Caputi, ${ }^{2}$ Amit Agarwal,,${ }^{1} *$ and Anna Cupolillo ${ }^{2, \dagger}$ \\ ${ }^{1}$ Department of Physics, Indian Institute of Technology Kanpur, Kanpur - 208016, India \\ ${ }^{2}$ Department of Physics, University of Calabria, via ponte Bucci, cubo 31/C 87036, Rende (CS) Italy \\ ${ }^{3}$ INFN, Sezione LNF, Gruppo Collegato di Cosenza, Cubo 31C, I-87036 Rende (CS), Italy \\ ${ }^{4}$ Electron Microscopy Facility, Istituto Italiano di Tecnologia, Via Morego 30, 16163 Genova, Italy \\ ${ }^{5}$ Department of Physics, National Cheng Kung University, 1 Ta-Hsueh Road 70101 Tainan, Taiwan \\ ${ }^{6}$ Istituto Italiano di Tecnologia-Graphene Labs via Morego, 3016163 Genova, Italy \\ ${ }^{7}$ Dipartimento di Scienze Fisiche e Chimiche (DSFC), \\ Universita dell'Aquila, Via Vetoio 10, I-67100 L'Aquila, Italy
}

(Dated: August 28, 2018)

\begin{abstract}
We explore the broadband excitation spectrum of bulk $\mathrm{PtTe}_{2}$ using electron energy loss spectroscopy and density functional theory. In addition to infrared modes related to intraband 3D Dirac plasmon and interband transitions between the 3D Dirac bands, we observe modes at 3.9, 7.5 and $19.0 \mathrm{eV}$ in the ultraviolet region. The comparison of the excitation spectrum with the calculated orbital-resolved density of states allows us to ascribe spectral features to transitions between specific electronic states. Additionally, we study the thickness dependence of the high-energy plasmon in the $\mathrm{PtTe}_{2}$ thin films. We show that, unlike graphene, the high-energy plasmon in $\mathrm{PtTe}_{2}$ thin film gets red-shifted by $\sim 2.5 \mathrm{eV}$ with increasing thickness.
\end{abstract}

\section{INTRODUCTION}

Recently, the $\mathrm{PtX}_{2}(\mathrm{X}=\mathrm{Se}, \mathrm{Te}, \mathrm{S})$ class of transitionmetal dichalcogenides (TMDCs) have attracted a huge interest of the scientific community. This class of TMDCs combines promising application capabilities along with the fundamental physics interest arising from the existence of topological type-II Dirac fermions. ${ }^{1-9}$ As opposed to type-I Dirac materials, which have a closed Fermi surface with either an electron or a hole pocket, type-II Dirac materials have an unbounded Fermi surface with both electron and hole pockets. ${ }^{3}$ The presence of bulk topological Dirac node forces the existence of massless surface states. This provides massless charge carriers with ultrahigh mobility confined at the surface plane. ${ }^{5}$

Thin layers of $\mathrm{PtX}_{2}$ class of materials are equally interesting because of their air stability, high mobility, and superior gas sensing properties, among others. ${ }^{2,10-14}$ The quantum confinement effect dramatically changes the properties of the thin layers of $\mathrm{PtTe}_{2}$ which undergo a metal-semiconductor transition with decreasing thickness. ${ }^{15,16}$ Apart from this, the monolayer exhibits a unique property: because of local dipole-induced Rashba effect, opposite spins with the same energy gets spatially separated on the opposite sides of the monolayer. ${ }^{17}$ This is known as spin layer locking and it can have potential applications in the electrically tunable spintronic devices.

Preceding discussions made it clear that $\mathrm{PtX}_{2}$ class of materials has emerged as a promising material for the future electronics. While the band structure of $\mathrm{PtTe}_{2}$ has been explored comprehensively ${ }^{5}$, along with the Dirac plasmons (collective density excitations) in the infrared range of the electromagnetic spectrum ${ }^{18}$, the high-energy excitations in $\mathrm{PtTe}_{2}$ still remain unexplored. The comprehension of the excitation spectrum of collective modes in the visible-ultraviolet is crucial to de- vise broadband photodetectors ${ }^{19,20}$, ultraviolet-imaging applications $^{21}$ and broadband plasmonic devices. ${ }^{22,23}$ Monolayer $\mathrm{PtTe}_{2}$ has the smallest energy band gap in the $\mathrm{PtX}_{2}$ class of materials ${ }^{24}$, offering it an advantage over the other members for applications in nano-electronics.

Motivated by this, in this paper we probe the broadband excitation spectrum of bulk crystals and thin layers of $\mathrm{PtTe}_{2}$, using electron energy loss spectroscopy $(\text { EELS })^{25}$ complemented by detailed ab-initio calculations. EELS probes the broadband dielectric response of the system to a negatively charged probe, allowing for spectral contributions from both plasmonic modes and non-vertical transitions from valence-band to conductionband electronic states. Therefore, EELS investigations supplement the studies of the absorption and emission processes of TMDCs in the long-wavelength limit ${ }^{26}$ involving only vertical transitions from occupied to unoccupied states. The nature of various experimentally probed EELS excitations is identified by means of calculations of the band-structure and the loss function based on density-functional theory (DFT). In addition to the bulk excitation spectrum, we also explore the changes in the broadband excitation spectrum of $\mathrm{PtTe}_{2}$ on reducing its thickness to few layers. Contrarily to the case of graphene and phosphorene, a blue-shift of the highenergy plasmon frequency is observed for thin layers compared to the bulk. ${ }^{27-29}$

The manuscript is organized as follows: In Sec. II we briefly describe the crystal structure, experimental details related to the growth and characterization of $\mathrm{PtTe}_{2}$ along with that of the reflection EELS. This is followed by a discussion of the theoretical ab-initio calculation of the EELS spectrum and other computational details in Sec. III. The broadband EELS spectrum of bulk $\mathrm{PtTe}_{2}$ is discussed in Sec. IV, followed by the discussion of the excitation spectrum of thin layers in Sec. V. Finally, we 
(a)

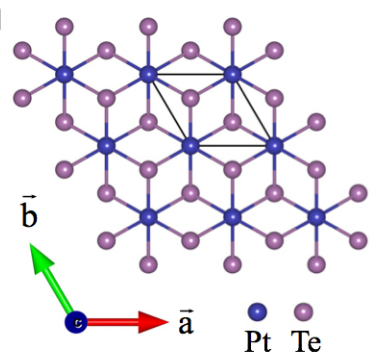

(d)

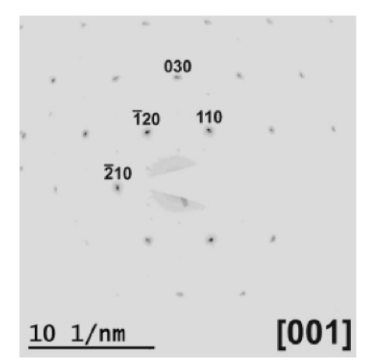

(b)

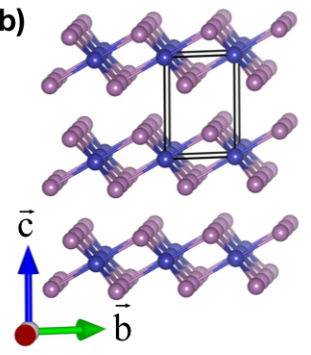

(e)

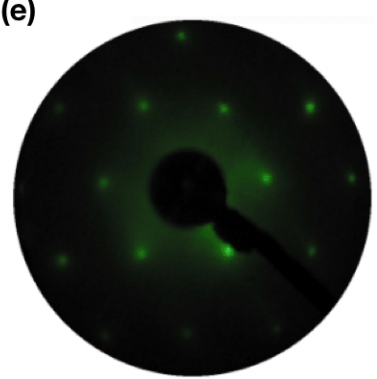

(c)

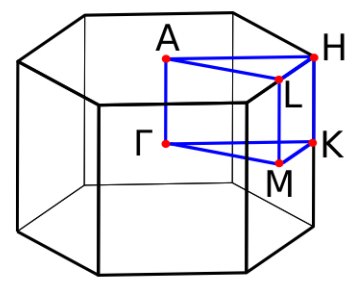

(f)

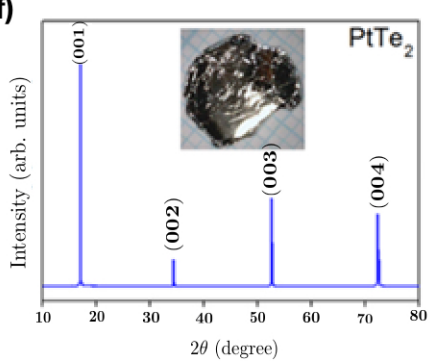

FIG. 1. Panels (a) and (b) show the top and side view of the atomic crystal structure of PtTe 2 . The corresponding bulk Brillouin zone of the hexagonal $\mathrm{PtTe}_{2}$ crystal, along with various high symmetry points is shown in (c). (d) Selected area electron diffraction (SAED) patterns acquired on $\mathrm{PtTe}_{2}$ flakes match with [0001]-oriented single-crystal trigonal PtTe 2 (ICSD \#41373, moncheite). (e) LEED pattern of bulk $\mathrm{PtTe}_{2}$ single crystal oriented along the (0001) direction. (f) XRD pattern of (0001)-oriented planes of PtTe 2 . Only (00n) peaks are observed.

summarize our findings in Sec. VI.

\section{GROWTH, CRYSTAL STRUCTURE, CHARACTERIZATION AND DETAILS OF EELS}

\section{A. Growth}

Single crystals of $\mathrm{PtTe}_{2}$ were prepared by the self-flux method. High-purity Pt (99.99\%) foil and Te ingot $(99.9999 \%)$ were mixed in the ratio of $1: 17$ and wrapped in a quartz tube under vacuum. The quartz tube was heated to $1000{ }^{\circ} \mathrm{C}$, dwelled there for 8 hours, and slowly cooled at a rate of $3-5{ }^{\circ} \mathrm{C} / \mathrm{h}$ to $500{ }^{\circ} \mathrm{C}$. Successively, the excess Te flux was separated by centrifugation. The resulting crystals have typical dimensions of $8 \times 8 \times 1 \mathrm{~mm}^{11}$ with the c-axis perpendicular to the plates and can be easily cleaved. The structure of the grown crystals was examined by X-ray diffraction (Bruker D2 PHASER) using $\mathrm{Cu} \mathrm{K}_{\alpha}$ radiation and Laue diffraction at room temperature.

Thin flakes of $\mathrm{PtTe}_{2}$ were obtained by liquid-phase exfoliation of bulk $\mathrm{PtTe}_{2}$ in N-methylpyrrolidone (NMP).

\section{B. Crystal Structure}

Bulk $\mathrm{PtTe}_{2}$ belongs to the large family of $1 \mathrm{~T}$-metal dichalcogenides with $\mathrm{CdI}_{2}$ type crystal structure [space group $\mathrm{P} \overline{3} \mathrm{~m} 1(164)]$. The bulk structure can be viewed as a collection of isolated monolayers stacked in the out of plane direction - see Fig. 1(a) and 1(b). In each of these monolayers there are three sublayers, Te-Pt-Te, where the central Pt atom is strongly bonded with six neighbouring Te atoms forming a hexagonal honeycomb structure. Both selected area electron diffraction (SAED) and low-energy electron diffraction (LEED) patterns shown in Fig. 1(d) and 1(e) match with [0001]-oriented flakes and bulk $\mathrm{PtTe}_{2}$, respectively. Correspondingly, the XRD pattern only exhibits (00n) peaks as shown in Fig. 1(f).

\section{Characterization}

To demonstrate cleanliness, the surface has been characterized by means of vibrational spectroscopy and X-ray photoelectron spectroscopy, without finding any contamination (see appendix B, and C). Once prepared in ultrahigh vacuum, the surface remains uncontaminated for a timescale of several weeks, thus ensuring sample stability. The low-energy electron diffraction (LEED) pattern shows sharp spots against a low background - see Fig. 1(e).

\section{Electron Energy Loss Spectroscopy}

The reflection EELS experiments were performed at room temperature by means of an EELS apparatus with two $50 \mathrm{~mm}$ hemispherical deflectors for both monochromator and analyzers, mounted in an ultra-high vacuum chamber at the University of Calabria, Italy. The pri- 
mary electron beam impinges on the sample with an incident angle $\theta_{i}$ of $43^{\circ}$ with respect to the surface normal, along the $\Gamma-K$ direction of the surface Brillouin zone. The primary electron beam energy is $E_{p}=100 \mathrm{eV}$.

SAED and STEM-EELS analyses were carried out at room temperature on a FEI Tecnai G2 F20 TWIN TEM, equipped with a Gatan Enfinium SE spectrometer at Istituto Italiano di Tecnologia, Genoa (Italy). For these experiments a primary electron beam energy $E_{p}=200$ $\mathrm{keV}$ and a collection angle of $13 \mathrm{mrad}$ were used. Samples for TEM analyses were prepared by drop casting of the flakes dispersion onto a holey carbon-coated $\mathrm{Cu}$ grid. The EELS spectra were collected from flakes regions suspended on holes in the carbon support film.

\section{THEORY}

\section{A. Energy Loss function}

The theoretical calculation of the electron energy loss function starts with the non-interacting density-density response function $\left(\chi_{\mathbf{G G}^{\prime}}^{0}\right)$ for a periodic lattice. It is obtained using the Adler-Wiser formula given by ${ }^{30,31}$,

$$
\begin{aligned}
& \chi_{\mathbf{G G}^{\prime}}^{0}(\mathbf{q}, \omega)=\frac{1}{\Omega} \sum_{\mathbf{k}}^{\mathrm{BZ}} \sum_{n, n^{\prime}} \frac{f_{n \mathbf{k}}-f_{n^{\prime} \mathbf{k}+\mathbf{q}}}{\omega+\epsilon_{n \mathbf{k}}-\epsilon_{n^{\prime} \mathbf{k}+\mathbf{q}}+i \eta} \times \\
& \left\langle\psi_{n \mathbf{k}}\left|e^{-i(\mathbf{q}+\mathbf{G}) \cdot \mathbf{r}}\right| \psi_{n^{\prime} \mathbf{k}+\mathbf{q}}\right\rangle_{\Omega_{\text {cell }}}\left\langle\psi_{n \mathbf{k}}\left|e^{i\left(\mathbf{q}+\mathbf{G}^{\prime}\right) \cdot \mathbf{r}^{\prime}}\right| \psi_{n^{\prime} \mathbf{k}+\mathbf{q}}\right\rangle_{\Omega_{\text {cell }}}
\end{aligned}
$$

The wave function $\psi_{n \mathbf{k}}$, eigenvalue $\epsilon_{n \mathbf{k}}$, and the corresponding Fermi-Dirac occupation function $f_{n \mathbf{k}}$ at wave vector $\mathbf{k}$ for the band with index $n$ are obtained from the ground-state calculations performed using DFT.

The interacting density-density response function can be obtained within the framework of time-dependent DFT (TDDFT) by solving a Dyson-like equation. Using a plane-wave basis for a periodic system, for a given $(\mathbf{q}, \omega)$, it can be expressed as

$$
\chi_{\mathbf{G G}^{\prime}}=\chi_{\mathbf{G G}^{\prime}}^{0}+\sum_{\mathbf{G}_{1}, \mathbf{G}_{\mathbf{2}}} \chi_{\mathbf{G G}_{\mathbf{1}}}^{0}(\mathbf{q}, \omega) K_{\mathbf{G}_{\mathbf{1}} \mathbf{G}_{\mathbf{2}}}(q) \chi_{\mathbf{G}_{\mathbf{2}} \mathbf{G}^{\prime}}(\mathbf{q}, \omega),
$$

where $\mathbf{G}$ and $\mathbf{q}$ are the reciprocal lattice vector and the Bloch wave vector, respectively, and $K_{\mathbf{G}_{1} \mathbf{G}_{\mathbf{2}}}$ is the interaction kernel, including both the Coulomb or Hartree term, as well as the exchange correlations.

Using the calculated $\chi_{\mathbf{G G}^{\prime}}$ matrix, the dielectric matrix, $\epsilon_{\mathbf{G G}^{\prime}}(\mathbf{q}, \omega)$, can obtained as,

$$
\epsilon_{\mathbf{G G}^{\prime}}^{-1}(\mathbf{q}, \omega)=\delta_{\mathbf{G G}^{\prime}}+\frac{4 \pi}{|\mathbf{q}+\mathbf{G}|^{2}} \chi_{\mathbf{G G}^{\prime}}(\mathbf{q}, \omega) .
$$

The macroscopic dielectric constant can be obtained from the dielectric matrix via the equation,

$$
\epsilon_{M}(\mathbf{q}, \omega)=\frac{1}{\epsilon_{00}^{-1}(\mathbf{q}, \omega)}
$$

The dynamical loss function, which is directly related to the experimental excitation spectrum probed by EELS, is now obtained from the macroscopic dielectric function,

$$
\mathcal{E}_{\mathrm{Loss}}(\mathbf{q}, \omega)=-\Im\left[\frac{1}{\epsilon_{M}(\mathbf{q}, \omega)}\right]
$$

Plasmons (collective density excitations) are characterized by the zeroes of the real part of the macroscopic dielectric function (the denominator of the density-density response function within RPA). Thus, they appear as peaks in the EELS spectrum. The calculations of the loss function are performed using the generalized RPA, which uses the local-field factors to add the impact of the exchange and correlation effects, in addition to the Hartree field ${ }^{32}$.

\section{B. Computational details}

Electronic band structure calculations were performed using a plane-wave basis set within the framework of DFT, as implemented in the VASP package. ${ }^{33,34} \mathrm{We}$ use the PAW pseudopotentials with $500 \mathrm{eV}$ kinetic energy cut-off for the plane-wave basis set. ${ }^{35}$ The exchangecorrelation part of the potential has been treated within the framework of generalized approximation scheme developed by Perdew-Burke-Ernzerhof. ${ }^{36}$ Starting from the experimental structure, we relax all the atomic positions until the forces on each atom are less than $0.001 \mathrm{eV} / \AA$. Spin-orbit interaction has been considered as a perturbation and treated in a self-consistent manner.

In order to calculate the response functions we use the GPAW code, which employs a real-space representation of the PAW potentials. ${ }^{37-39}$ A kinetic energy cutoff of 500 $\mathrm{eV}$ has been used for the plane-wave basis set. We use a $122 \times 122 \times 20 k$-grid to calculate the momentum dependence of the loss function. To incorporate the local-field effects, we use a plane-wave cutoff of $60 \mathrm{eV}$ which corresponds to 85 plane waves. A broadening parameter $\eta=0.05 \mathrm{eV}$ is assumed in all calculations of the response function.

\section{BROADBAND SPECTRUM OF BULK PtTe}

The experimental broadband EELS spectra of $\mathrm{PtTe}_{2}$ bulk sample, measured with an electron beam energy of $100 \mathrm{eV}$, is shown in Fig. 2(a). The broadband EELS spectrum shows distinctly resolved peaks at energies $\sim 0.5$, $\sim 1.4, \sim 3.9, \sim 7.5$ and $\sim 19.0 \mathrm{eV}$, among others. The lowest energy peak at $0.5 \mathrm{eV}$ is the intra-band gapped $3 \mathrm{D}$ Dirac plasmon excitation in bulk $\mathrm{PtTe}_{2}$, which disperses with the momentum [see Fig. 2(e)]. It has been discussed in detail in Ref. [18]. Here, we will focus on the remaining inter-band excitations, which are relatively less dispersive compared to the intra-band 3D Dirac plasmon peak. We find that their peak location and relative intensity is 
(a)

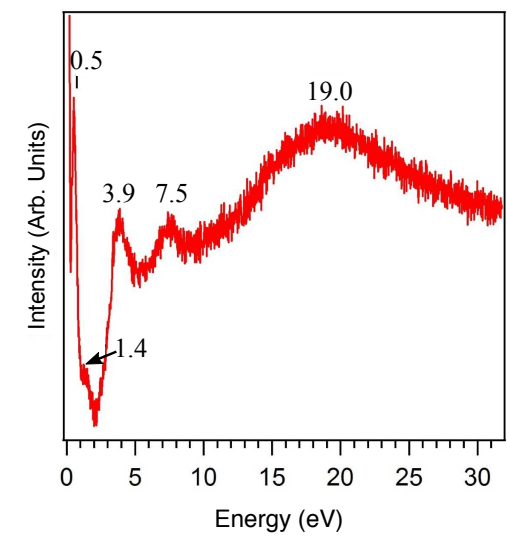

(d)

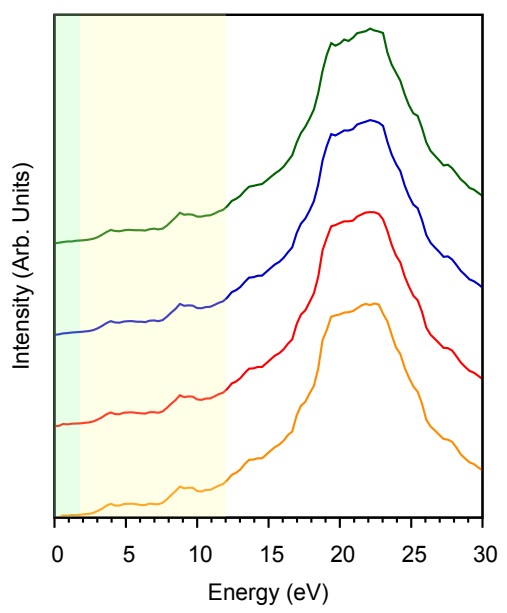

(b)

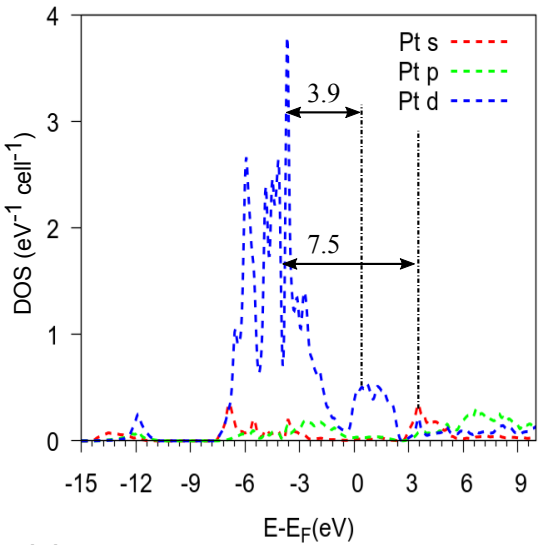

(e)

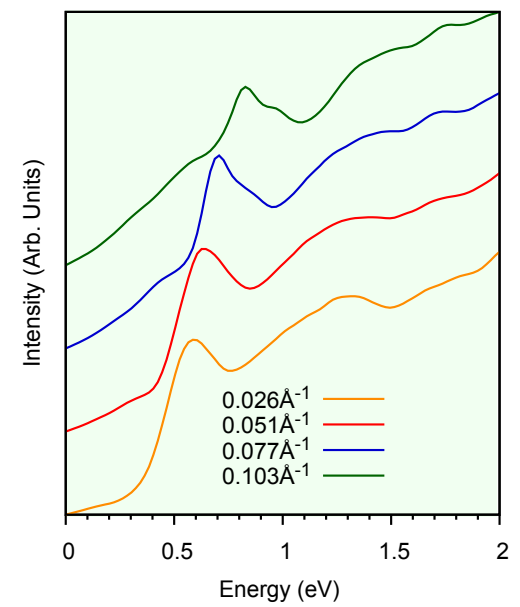

(c)

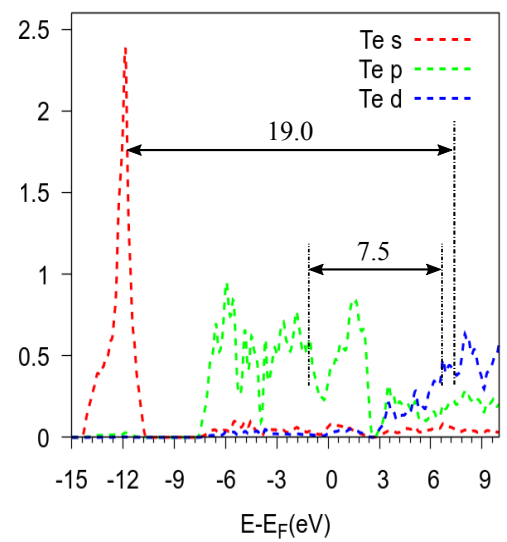

(f)

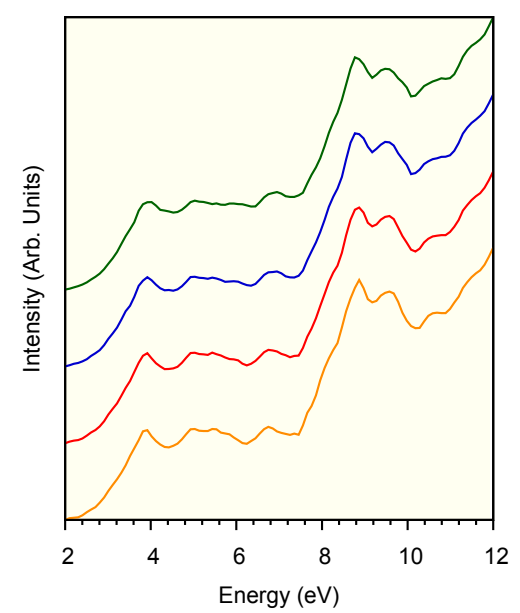

FIG. 2. (a) Broadband EELS spectrum for bulk PtTe 2 measured in reflection mode with a primary electron beam energy of 100 $\mathrm{eV}$. The EELS spectrum shows several distinct peaks at energies $0.5,1.4,3.9,7.5$ and $19.0 \mathrm{eV}$. The peak at $0.5 \mathrm{eV}$ is dispersive and it is associated with the intra-band density excitations ${ }^{18}$ in $\mathrm{PtTe}_{2}$. The other higher energy peaks correspond to inter-band transitions, which can be identified from the orbital-resolved DOS plot, shown in the panels (b) and (c). The corresponding orbital-resolved band-structure plots are shown in Fig. 3. The dominant transitions, corresponding to the observed EELS peaks, are marked by arrows. The experimental broadband EELS spectrum is also reasonably captured by the loss function obtained from ab-initio calculations, as shown in (d)-(f) for different momentum values, reported in the legend of panel (e). The $19.0 \mathrm{eV}$ peak in (d) has the highest intensity in the ab-initio calculations. The other dominant intra-band peaks at 3.9 and $7.5 \mathrm{eV}$ are resolved in panel (f).

nearly independent on scattering geometry and, consequently on the momentum in a momentum range of of $0.0-0.2 \AA^{-1}$.

In order to identify the inter-band transitions corresponding to the observed peaks in the EELS spectrum, we show the orbital-resolved density of states (DOS) in Fig. 2(b)-(c). The corresponding orbital-resolved band structure plot is shown in Fig. 3. The energetically lower valence band (VB) is situated approximately between 15 and $-10 \mathrm{eV}$ and it is mostly dominated by $\mathrm{Te}_{5 s}$ states. The upper VB extends from $-7 \mathrm{eV}$ up to the Fermi level and mostly comprises of $\mathrm{Pt}_{5 d}$ and $\mathrm{Te}_{5 p}$ orbitals. The lower conduction-band states till $\sim 3 \mathrm{eV}$ are also primarily formed by $\mathrm{Pt}_{5 d}$ and $\mathrm{Te}_{5 p}$ orbitals. The conduction- band states at higher energy have contributions mainly from the $\mathrm{Pt}_{6 p}$ and $\mathrm{Te}_{5 d}$ orbitals. Comparison of the orbital-resolved DOS with the observed spectral features in the broadband EELS spectrum allows us to identify the prominent states involved in the transitions. We find that the peaks at $3.9,7.5$ and $19.0 \mathrm{eV}$ are predominantly connected to $\mathrm{Pt}_{5 d} \rightarrow \mathrm{Pt}_{5 d} ; \mathrm{Te}_{5 p} \rightarrow \mathrm{Te}_{5 d}$; and $\mathrm{Te}_{5 s} \rightarrow$ $\mathrm{Te}_{5 d}$ transitions, respectively, as marked by arrows in Fig. 2(b)-(c). The theoretical loss function in Fig. 2(d)(f) also captures the qualitative features of the experimental excitation spectrum reasonably well.

It is worth reminding that EELS peaks cannot precisely match the energies of single-particle transitions observed with optical spectroscopies, as maxima in the loss 

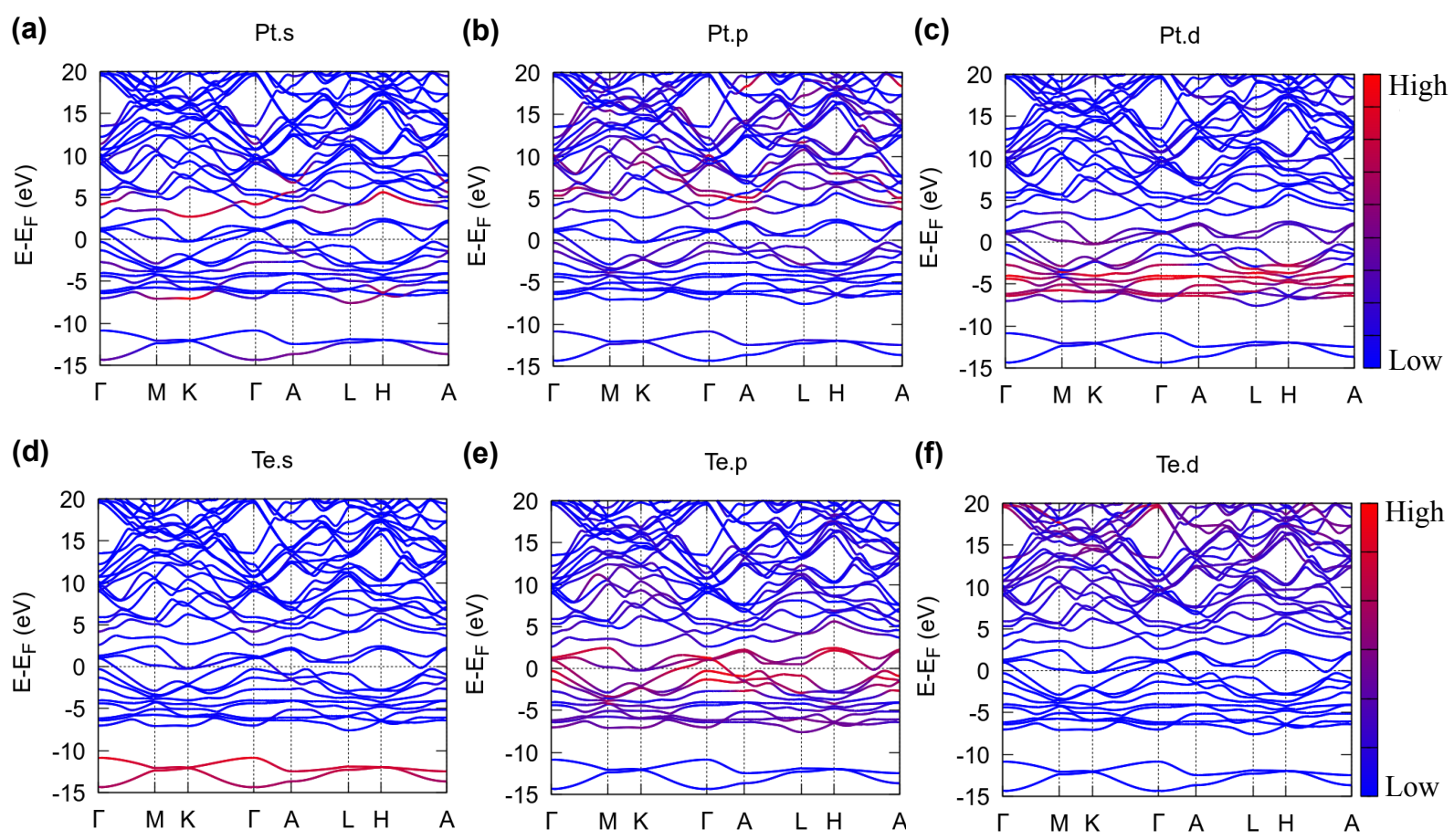

FIG. 3. Orbital-projected band structure of $\mathrm{PtTe}_{2}$. The lowest energy states are dominated by Te $\mathrm{T}_{s}$ orbital. Near the Fermi energy the states have major contributions from $\mathrm{Pt}_{5 d}$ and $\mathrm{Te}_{5 p}$ orbitals. The higher energy states are mostly $\mathrm{Pt}_{6 p}$ and $\mathrm{Te}_{5 d}$ states.

function are related to the maxima in $-\operatorname{Im}\left[1 / \epsilon_{M}(\omega)\right]$. Conversely, optical transitions are identified with maxima in $\operatorname{Im}\left[\epsilon_{M}(\omega)\right]^{40}$, that are actually shifted with respect to the maxima of $-\operatorname{Im}\left[1 / \epsilon_{M}(\omega)\right]^{41}$. Having discussed the EELS spectrum of the bulk PtTe ${ }_{2}$ crystal, we now proceed to discuss the EELS spectrum of thin $\mathrm{PtTe}_{2}$ layers in the next section.

\section{BROADBAND SPECTRUM OF THIN PtTe LAYERS}

In this section, we explore the thickness dependence of the broadband EELS spectrum of $\mathrm{PtTe}_{2}$ thin films. To this aim, we have employed the STEM-EELS technique, which affords the spatial resolution necessary to identify regions with different thickness within the flakes. As shown in Fig. 4(a), the liquid-phase exfoliation of $\mathrm{PtTe}_{2}$ has produced flakes with inhomogeneous thickness. Therefore, for STEM-EELS experiments different thicknesses were probed on different areas of same fragments.

Previously, the thickness dependence of the high energy EELS peaks has been done for the case of $\sigma+\pi$ plasmons of graphene. In graphene, the plasmon energy shifts by about $10 \mathrm{eV}$ when going from monolayer graphene to multilayers. ${ }^{27,28,42}$ Remarkably, a similar blue-shift has been detected by both reflection EELS in graphene layers epitaxially grown on silicon carbide ${ }^{42}$, and by EELS-TEM in free-standing graphene flakes exfoliated from graphite. ${ }^{27,28}$ This is irrespective of the dissimilarities in the plasmon energy in the monolayer regime $\left(10 \mathrm{eV}\right.$ in the latter case $\mathrm{e}^{27,28}$ and $14.6 \mathrm{eV}$ in the former one $\mathrm{e}^{42}$ ). This blue-shift of the plasmon energy in graphene with increasing thickness had been ascribed to the effect of interlayer Coulomb coupling ${ }^{28}$. In contrast to graphene, the high energy plasmon peak in phosphorene does not show any thickness dependence ${ }^{43}$.

Our STEM-EELS investigation suggests that in $\mathrm{PtTe}_{2}$ the plasmon band changes in both line-shape and energy position as a function of thickness. Specifically, a symmetrical line-shape, centered at about $23 \mathrm{eV}$ (Fig. 4c), is recorded for thinner flakes, whereas an asymmetrical line-shape, with a corresponding centroid around 20.5 $\mathrm{eV}$, are revealed for thicker flakes. Thus, in contrast to graphene $^{27,28}$, and phosphorene ${ }^{43}$ the high energy plasmon peak in $\mathrm{PtTe}_{2}$ is red-shifted with increasing thickness. However, the layer-dependent plasmon-energy shift in $\mathrm{PtTe}_{2}$ is much lower $(\sim 2.5 \mathrm{eV})$ than that of graphene $(\sim 10 \mathrm{eV})$. The change in the line shape, with an asymmetric shape characterizing the thinner regions, is ascribable to the multiple contributions (i.e., due to regions with different thickness) to the spectra acquired in the thinner regions of the flakes. The presence of contributions from regions few $\mathrm{nm}$ away from the position of the electron beam is due to the delocalization of inelastic scattering, prominent in the low energy-loss range ${ }^{44,45}$. 

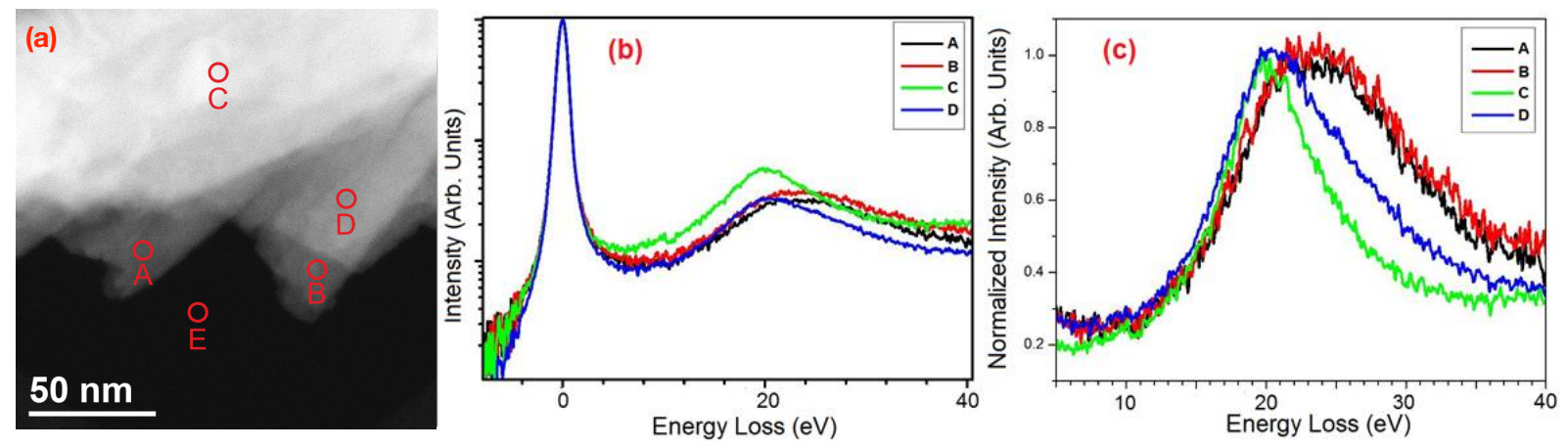

FIG. 4. (a) HAADF-STEM image of the fragmented PtTe 2 flake suspended on a hole in the amorphous carbon support film used for STEM-EELS analysis. The points A and B exhibit a lower thickness (lower brightness in HAADF-STEM mode) than point $\mathrm{D}$, while the point $\mathrm{C}$ has the highest thickness in the flake. Only the zero-loss peak appears in the spectrum recorded at point E, i.e. $20 \mathrm{~nm}$ far from the $\mathrm{PtTe}_{2}$ flake. (b) The broadband STEM-EELS spectra acquired for points A, B, C and D. In panel (b) the intensity is normalized to the zero-loss peak maximum, while in panel (c) it is normalized to that of the peak at $\sim 20 \mathrm{eV}$, in order to highlight the dissimilarities in the line-shape of the peaks corresponding to different thickness.

\section{CONCLUSIONS}

We have probed the broadband excitation spectrum of bulk crystals and thin layers of $\mathrm{PtTe}_{2}$, using EELS in reflection mode for bulk and STEM-EELS for thin layers. In the case of bulk $\mathrm{PtTe}_{2}$ we find different modes in the ultraviolet regime at $3.9,7.5$ and $19.0 \mathrm{eV}$, in addition to the excitations associated to the 3D Dirac cones observed in the infrared range at 0.5 and $1.4 \mathrm{eV}$. These observations are well explained by the DFT-based orbitalresolved band-structure and DOS calculations. Specifically, we find that in bulk $\mathrm{PtTe}_{2}$ the observed peaks at $3.9,7.5$ and $19.0 \mathrm{eV}$ are predominantly connected to $\mathrm{Pt}_{5 d} \rightarrow \mathrm{Pt}_{5 d} ; \mathrm{Te}_{5 p} \rightarrow \mathrm{Te}_{5 d} ;$ and $\mathrm{Te}_{5 s} \rightarrow \mathrm{Te}_{5 d}$ transitions, respectively. In thin layers, with decreasing thickness, the high-energy plasmon peak gets shifted from 20.5 to $23.0 \mathrm{eV}$. This red-shift with increasing thickness is in contrast to the blue-shift observed in case of graphene. Moreover, with increasing number of layers an increase in the anisotropy of the line-shape of the high-energy plasmon peak is observed. This peculiarity can be exploited for characterizing the thickness of $\mathrm{PtTe}_{2}$ thin films. Similar physics is expected to play out in other members of the family, including $\mathrm{PdTe}_{2}$ and $\mathrm{PtSe}_{2}$, among others.

\section{Appendix A: Valence-band states in $\mathrm{PtTe}_{2}$}

As an additional check we explicitly check that there are no valence-band states below the $-14 \mathrm{eV}$ in $\mathrm{PtTe}_{2}$, by calculating the band-structure over a wide energy range. The calculated band-structure is shown in Fig. 5: it is evident that there are no valence-band states in $\mathrm{PtTe}_{2}$ from approximately -14 to $-50 \mathrm{eV}$.

\section{Appendix B: Surface chemical reactivity of $\mathrm{PtTe}_{2}$ single crystals}

To evaluate the surface chemical reactivity of $\mathrm{PtTe}_{2}$, we have carried out high-resolution X-ray photoelectron spectroscopy (XPS) experiments (Fig. 6). We addressed firstly the evolution of $\mathrm{Pt} 4 \mathrm{f}$ and $\mathrm{Te} 3 \mathrm{~d}$ core levels upon several treatments $\left(\mathrm{O}_{2}\right.$ dosage and air exposure), with respect to spectra measured for pristine $\mathrm{PtTe}_{2}$. From the analysis of Te 3d core-level spectra (Fig. 6), we deduce that the as-cleaved undefected $\mathrm{PtTe}_{2}$ surface is inert to oxygen exposure. In fact, only $3 \mathrm{~d}_{5 / 2}$ and $3 \mathrm{~d}_{3 / 2}$ core levels at the binding energies of 573.1 and $583.5 \mathrm{eV}$, corresponding to an oxidation state Te(0), are observed in Fig. 6 for both the pristine $\mathrm{PtTe}_{2}$ (red curve) and the same surface exposed to a dose of $10^{6} \mathrm{~L}\left(1 \mathrm{~L}=1.33 \times 10^{-6}\right.$ mbars $)$ of

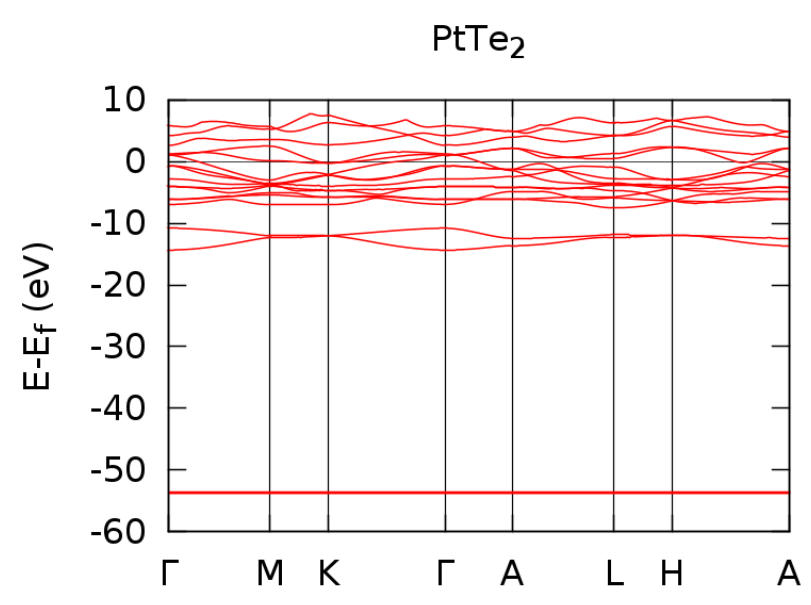

FIG. 5. Band structure of $\mathrm{PtTe}_{2}$ over a wide energy region. Evidently, there are no energy states in between $-13 \mathrm{eV}$ to -50 $\mathrm{eV}$. 


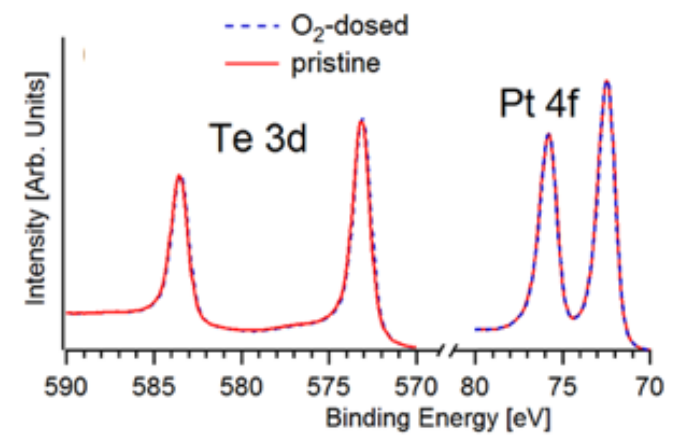

FIG. 6. High-resolution XPS spectra of Pt-4f and Te-3d core levels taken for the pristine $\mathrm{PtTe}_{2}$ sample (red curve) and the same surface exposed to $10^{6} \mathrm{~L}$ of $\mathrm{O}_{2}$ (dashed blue curve). For all spectra, the photon energy is $745 \mathrm{eV}$ and the energy resolution is $0.1 \mathrm{eV}$.

$\mathrm{O}_{2}$ at room temperature (dashed blue curve).

The $\mathrm{Pt}$ ff doublet reported in Fig. 6 is observed at binding energies of $72.5\left(4 \mathrm{f}_{7 / 2}\right)$ and $75.9\left(4 \mathrm{f}_{5 / 2}\right) \mathrm{eV}$, respectively. Similar values of the binding energy for Pt-4f core levels have been reported for $\mathrm{PtSe}_{2}{ }^{14,46}$.

To further assess the surface chemical reactivity in $\mathrm{PtTe}_{2}$-based systems, we have also carried out vibrational experiments by means of high-resolution electron energy loss spectroscopy (HREELS). Explicitly, we have exposed pristine $\mathrm{PtTe}_{2}$ to water and oxygen. The corresponding vibrational spectra are reported in Fig. 7. For the case of pristine (undefected) $\mathrm{PtTe}_{2}$, the vibrational spectrum remains featureless even after exposure of water and oxygen at room temperature (Fig. 7). Likewise, no vibrational peaks are revealed in the air-exposed undefected $\mathrm{PtTe}_{2}$ surface (red curve in Fig. 7). Combined with the XPS results reported in Fig. 6, these data lead to the conclusion that undefected $\mathrm{PtTe}_{2}$ does not react at room temperature with ambient gases. This finding has a particular interest in view of applications in optoelectronics based on $\mathrm{PtTe}_{2}$. *amitag@iitk.ac.in

† anna.cupolillo@fis.unical.it

1 Andres Castellanos-Gomez, "Why all the fuss about 2d semiconductors?" Nature Photonics 10, 202 (2016).

${ }^{2}$ Liang Li, Weike Wang, Yang Chai, Huiqiao Li, Mingliang Tian, and Tianyou Zhai, "Few layered $\mathrm{pts}_{2}$ phototransistor on hbn with high gain," Advanced Functional Materials 27, 1701011 (2017)

3 Alexey A. Soluyanov, Dominik Gresch, Zhijun Wang, QuanSheng Wu, Matthias Troyer, Xi Dai, and B. Andrei Bernevig, "Type-ii weyl semimetals," Nature 527, 495 EP - (2015).

4 Mingzhe Yan, Huaqing Huang, Kenan Zhang, Eryin Wang, Wei Yao, Ke Deng, Guoliang Wan, Hongyun Zhang, Masashi Arita, Haitao Yang, Zhe Sun, Hong Yao, Yang Wu, Shoushan Fan, Wenhui Duan, and Shuyun Zhou, "Lorentz-violating type-ii dirac fermions in transition metal dichalcogenide ptte2," Nature Communications 8, 257 (2017).

${ }^{5}$ M. S. Bahramy, O. J. Clark, B.-J. Yang, J. Feng, L. Bawden, J. M. Riley, I. Markovic, F. Mazzola, V. Sunko, D. Biswas, S. P. Cooil, M. Jorge, J. W. Wells, M. Leandersson, T. Balasubramanian, J. Fujii, I. Vobornik, J. E. Rault, T. K. Kim, M. Hoesch, K. Okawa, M. Asakawa, T. Sasagawa, T. Eknapakul, W. Meevasana, and P. D. C. King, "Ubiquitous formation of bulk dirac cones and topological surface states from a single orbital manifold in transition-metal dichalcogenides," Nature Materials 17, 21 (2017).

6 Han-Jin Noh, Jinwon Jeong, En-Jin Cho, Kyoo Kim, B. I. Min, and Byeong-Gyu Park, "Experimental realization of type-ii dirac fermions in a pdte ${ }_{2}$ superconductor," Phys. Rev. Lett. 119, 016401 (2017).

7 Kenan Zhang, Mingzhe Yan, Haoxiong Zhang, Huaqing Huang, Masashi Arita, Zhe Sun, Wenhui Duan, Yang Wu, and Shuyun Zhou, "Experimental evidence for type-ii dirac semimetal in ptse ${ }_{2}$," Phys. Rev. B 96, 125102 (2017).

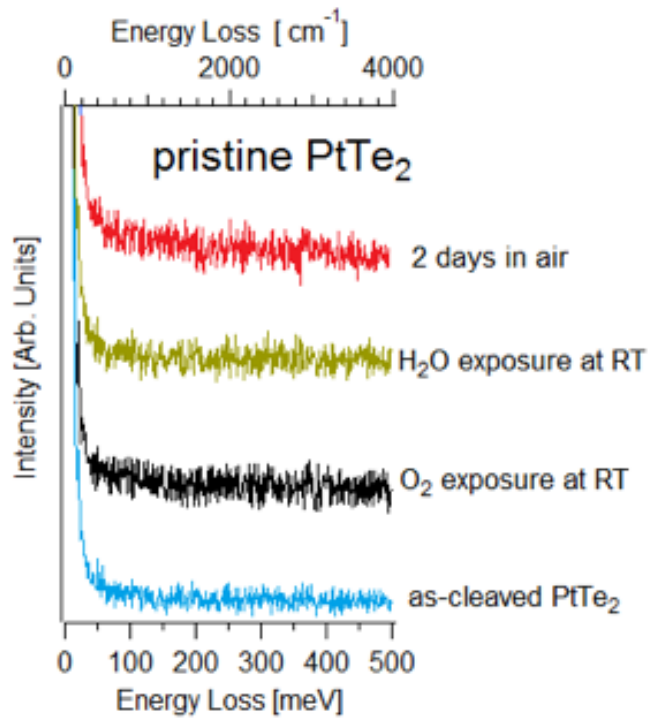

FIG. 7. Vibrational spectra for as-cleaved $\mathrm{PtTe}_{2}$ and for the same surface exposed to $10^{3} \mathrm{~L}$ of $\mathrm{O}_{2}$ and $\mathrm{H}_{2} \mathrm{O}$ at room temperature. Successively, the surface has been exposed to air. No vibrational peak is observed.

8 Huaqing Huang, Shuyun Zhou, and Wenhui Duan, "Typeii dirac fermions in the ptse $_{\mathbf{2}}$ class of transition metal dichalcogenides," Phys. Rev. B 94, 121117 (2016).

9 Tay-Rong Chang, Su-Yang Xu, Daniel S. Sanchez, WeiFeng Tsai, Shin-Ming Huang, Guoqing Chang, ChuangHan Hsu, Guang Bian, Ilya Belopolski, Zhi-Ming Yu, Shengyuan A. Yang, Titus Neupert, Horng-Tay Jeng, Hsin Lin, and M. Zahid Hasan, "Type-ii symmetryprotected topological dirac semimetals," Phys. Rev. Lett. 119, 026404 (2017). 
10 X. Lin et. al., "Intrinsically patterned two-dimensional materials for selective adsorption of molecules and nanoclusters," Nat Mater 16, 717-721 (2017).

11 Yuda Zhao, Jingsi Qiao, Zhihao Yu, Peng Yu, Kang Xu, Shu Ping Lau, Wu Zhou, Zheng Liu, Xinran Wang, Wei $\mathrm{Ji}$, and Yang Chai, "High-electron-mobility and air-stable 2d layered ptse2 fets," Advanced Materials 29, 1604230 (2017).

12 Xinyi Chia, Ambrosi Adriano, Petr Lazar, Zdenk Sofer, Jan Luxa, and Martin Pumera, "Layered platinum dichalcogenides (pts2, ptse2, and ptte2) electrocatalysis: Monotonic dependence on the chalcogen size," Advanced Functional Materials 26, 1505402 (2016).

13 Y Wang et. al., "Monolayer ptse2, a new semiconducting transition-metal-dichalcogenide, epitaxially grown by direct selenization of pt," Nano Letters 15, 4013-4018 (2015).

14 Zegao Wang, Qiang Li, Flemming Besenbacher, and Mingdong Dong, "Facile synthesis of single crystal ptse2 nanosheets for nanoscale electronics," Advanced Materials 28, 10224-10229 (2016).

15 Huifang Ma, Peng Chen, Bo Li, Jia Li, Ruoqi Ai, Zhengwei Zhang, Guangzhuang Sun, Kangkang Yao, Zhaoyang Lin, Bei Zhao, Ruixia Wu, Xuwan Tang, Xidong Duan, and Xiangfeng Duan, "Thickness-tunable synthesis of ultrathin type-ii dirac semimetal ptte 2 single crystals and their thickness-dependent electronic properties," Nano Letters 18, 3523-3529 (2018).

16 Alberto Ciarrocchi, Ahmet Avsar, Dmitry Ovchinnikov, and Andras Kis, "Thickness-modulated metalto-semiconductor transformation in a transition metal dichalcogenide," Nature Communications 9, 919 (2018).

17 Wei Yao, Eryin Wang, Huaqing Huang, Ke Deng, Mingzhe Yan, Kenan Zhang, Koji Miyamoto, Taichi Okuda, Linfei Li, Yeliang Wang, Hongjun Gao, Chaoxing Liu, Wenhui Duan, and Shuyun Zhou, "Direct observation of spin-layer locking by local rashba effect in monolayer semiconducting ptse2 film," Nature Communications 8, 14216 (2017).

18 Antonio Politano, Gennaro Chiarello, Barun Ghosh, Krishanu Sadhukhan, Chia-Nung Kuo, Chin Shan Lue, Vittorio Pellegrini, and Amit Agarwal, "3d dirac plasmons in the type-ii dirac semimetal ptte ${ }_{2}$," Phys. Rev. Lett. 121, 086804 (2018).

19 F. H. L. Koppens, T. Mueller, Ph Avouris, A. C. Ferrari, M. S. Vitiello, and M. Polini, "Photodetectors based on graphene, other two-dimensional materials and hybrid systems," Nature Nanotechnology 9, 780 (2014).

20 Xuechao Yu, Peng Yu, Di Wu, Bahadur Singh, Qingsheng Zeng, Hsin Lin, Wu Zhou, Junhao Lin, Kazu Suenaga, Zheng Liu, and Qi Jie Wang, "Atomically?thin noble metal dichalcogenide: a broadband mid-infrared semiconductor," Nature Communications 9, 1545 (2018).

21 X. Zhang, K. Wang, J. Ma, Q. Zhang, P. Yan, and X. Tian, "Ultraviolet imaging based on surface plasmon resonance with azo-polymer sensing layer," IEEE Photonics Technology Letters 27, 1297-1300 (2015).

22 Jun-Yu Ou, Jin-Kyu So, Giorgio Adamo, Azat Sulaev, Lan Wang, and Nikolay I. Zheludev, "Ultraviolet and visible range plasmonics in the topological insulator bi1.5sb0.5te1.8se1.2," Nature Communications 5, 5139 (2014).

23 Francesco Bisio, Remo Proietti Zaccaria, Riccardo Moroni, Giulia Maidecchi, Alessandro Alabastri, Grazia Gonella, Angelo Giglia, Laura Andolfi, Stefano Nannarone, Lorenzo
Mattera, and Maurizio Canepa, "Pushing the high-energy limit of plasmonics," ACS Nano 8, 9239-9247 (2014).

24 San-Dong Guo and Jian-Li Wang, "Spinorbital coupling effect on the power factor in semiconducting transitionmetal dichalcogenide monolayers," Semiconductor Science and Technology 31, 095011 (2016).

25 M. Rocca, "Low-energy eels investigation of surface electronic excitations on metals," Surface Science Reports 22, $1-71$ (1995).

${ }^{26}$ Hualing Zeng and Xiaodong Cui, "An optical spectroscopic study on two-dimensional group-vi transition metal dichalcogenides," Chem. Soc. Rev. 44, 2629-2642 (2015).

27 T. Eberlein, U. Bangert, R. R. Nair, R. Jones, M. Gass, A. L. Bleloch, K. S. Novoselov, A. Geim, and P. R. Briddon, "Plasmon spectroscopy of free-standing graphene films," Phys. Rev. B 77, 233406 (2008).

28 P. Wachsmuth, R. Hambach, G. Benner, and U. Kaiser, "Plasmon bands in multilayer graphene," Phys. Rev. B 90, 235434 (2014).

29 Barun Ghosh, Piyush Kumar, Anmol Thakur, Yogesh Singh Chauhan, Somnath Bhowmick, and Amit Agarwal, "Anisotropic plasmons, excitons, and electron energy loss spectroscopy of phosphorene," Phys. Rev. B 96, 035422 (2017).

30 Stephen L. Adler, "Quantum theory of the dielectric constant in real solids," Phys. Rev. 126, 413-420 (1962).

31 Nathan Wiser, "Dielectric constant with local field effects included," Phys. Rev. 129, 62-69 (1963).

32 G. Giuliani and G. Vignale, Quantum Theory of the Electron Liquid, Masters Series in Physics and Astronomy (Cambridge University Press, 2005).

33 W. Kohn and L. J. Sham, "Self-consistent equations including exchange and correlation effects," Phys. Rev. 140, A1133-A1138 (1965).

34 G. Kresse and J. Furthmüller, "Efficient iterative schemes for ab initio total-energy calculations using a plane-wave basis set," Phys. Rev. B 54, 11169-11186 (1996).

35 G. Kresse and D. Joubert, "From ultrasoft pseudopotentials to the projector augmented-wave method," Phys. Rev. B 59, 1758-1775 (1999).

36 John P. Perdew, Kieron Burke, and Matthias Ernzerhof, "Generalized gradient approximation made simple," Phys. Rev. Lett. 77, 3865-3868 (1996).

37 J. J. Mortensen, L. B. Hansen, and K. W. Jacobsen, "Realspace grid implementation of the projector augmented wave method," Phys. Rev. B 71, 035109 (2005).

38 J. Enkovaara et. al., "Electronic structure calculations with gpaw: a real-space implementation of the projector augmented-wave method," Journal of Physics: Condensed Matter 22, 253202 (2010).

39 S. R. Bahn and K. W. Jacobsen, "An object-oriented scripting interface to a legacy electronic structure code," Comput. Sci. Eng. 4, 56-66 (2002).

40 Frederick Wooten, Optical Properties of Solids, (1972), 10.1016/B978-0-12-763450-0.50001-5.

41 Ansgar Liebsch, Electronic excitations at metal surfaces (Springer Science \& Business Media, 2013).

42 Jiong Lu, Kian Ping Loh, Han Huang, Wei Chen, and Andrew T. S. Wee, "Plasmon dispersion on epitaxial graphene studied using high-resolution electron energy-loss spectroscopy," Phys. Rev. B 80, 113410 (2009).

43 Ryan J. Wu, Mehmet Topsakal, Tony Low, Matthew C. Robbins, Nazila Haratipour, Jong Seok Jeong, Renata M. Wentzcovitch, Steven J. Koester, and K. Andre Mkhoyan, 
"Atomic and electronic structure of exfoliated black phosphorus," Journal of Vacuum Science \& Technology A 33, 060604 (2015).

44 C.C. Ahn, Transmission electron energy loss spectrometry in materials science and the EELS atlas (Wiley-VCH, 2004).

45 R.F. Egerton, Electron Energy-Loss Spectroscopy in the Electron Microscope, Language of science (Plenum Press,
1996).

46 Chanyoung Yim, Kangho Lee, Niall McEvoy, Maria OBrien, Sarah Riazimehr, Nina C. Berner, Conor P. Cullen, Jani Kotakoski, Jannik C. Meyer, Max C. Lemme, and Georg S. Duesberg, "High-performance hybrid electronic devices from layered ptse 2 films grown at low temperature," ACS Nano 10, 9550-9558 (2016). 